\title{
A PROBLEM ON CYCLIC SUBGROUPS OF FINITE GROUPS
}

\author{
by THOMAS J. LAFFEY \\ (Received 5th June 1972)
}

\section{Introduction}

Let $G$ be a finite group and let $S$ be a subgroup of $G$ with

$$
\operatorname{core}(S)=\bigcap_{x \in G} x^{-1} S x=1 \text {. }
$$

We say $(G, S)$ has property $\left({ }^{*}\right)$ if there exists $x \in G$ such that $S \cap x^{-1} S x=1$. A question which immediately arises is the following; what conditions on $G, S$. ensure that $(G, S)$ has property $\left({ }^{*}\right)$ ?

It has been shown by J. S. Brodkey (1) that $(G, S)$ has property $\left({ }^{*}\right)$ if $S$ is an abelian Sylow $p$-subgroup of $G$ for some prime $p$. Brodkey's argument can easily be extended to the case where $S$ is an abelian Hall subgroup of the group $G$. (See also (2).)

It has been shown by N. Ito (3) that if $G$ is soluble and $S$ is a Sylow $p$ subgroup of $G$, then $(G, S)$ has property $\left(^{*}\right)$ except possibly when $p=2$ or $p$ is a Mersenne prime.

In this note we consider the case where $S$ is cyclic. We show that $(G, S)$ has property $\left({ }^{*}\right)$ if $G$ is simple and that if $G$ is soluble and $S \cap F(G)=1$, then $(G, S)$ has property $(*)$.

Our results suggest that $(G, S)$ has property $\left(^{*}\right)$ if $S$ is cyclic and $S \cap F(G)=1$, but we have not been able to prove this in general.

The notation is standard. We recall that if $G$ is a finite group $F(G)$ denotes the maximal nilpotent normal subgroup of $G$.

\section{Preliminary results}

Lemma 1. Let $q, p_{1}, \ldots, p_{n}$ be distinct prime numbers. For each $i$ let $a_{i}$ be the least positive integer for which $q^{a_{i}}=1 \bmod p_{i}$. Then $\sum_{i=1}^{n}\left(1 / q^{a_{i}}\right)<1-1 / q$.

Proof. Let $w(m)$ denote the number of distinct prime divisors of the positive integer $m$.

Then

$$
\begin{aligned}
\sum_{i=1}^{n}\left(1 / q^{a_{t}}\right) & <\sum_{i=1}^{\infty} \frac{w\left(q^{i}-1\right)}{q^{i}}<\sum_{i=1}^{\infty} \frac{\log _{2} q^{i}}{q^{i}} \\
& =q \log _{2} q /(q-1)^{2} .
\end{aligned}
$$

This implies the result for $q \geqq 5$.

E.M.S. -18/4-A 
Next

Finally

$$
\sum_{i=1}^{n}\left(\frac{1^{a}}{3}\right)<\frac{1}{3}+\frac{1}{27}+\sum_{i=3}^{\infty} \frac{i}{3^{i}}<\frac{2}{3}
$$

$$
\sum_{i=1}^{n}\left(\frac{1}{2} a^{i}\right)<\frac{1}{4}+\frac{1}{8}+\frac{1}{16}+\frac{1}{32}+\frac{1}{128}+\frac{1}{256}+\sum_{i=9}^{\infty}\left(i / 2^{i}\right)<\frac{1}{2} .
$$

Lemma 2. Let $q_{1}<q_{2}<q_{3} \ldots$ be the sequence of prime numbers. Then

$$
\sum_{i=1}^{\infty}\left(1 / q_{i}^{2}\right)<\frac{2}{3}
$$

Proof. The result follows immediately from the fact that

$$
\sum_{n=1}^{\infty}\left(1 / n^{2}\right)=\pi^{2} / 6
$$

\section{Cyclic group action on a nilpotent group}

This section is devoted to a proof of the following result:

Theorem 1. Let $Q$ be a finite nilpotent group and let $A$ be a cyclic group of automorphisms of $Q$. Then there exists $v \in Q$ such that $v a \neq v$ for any $a \neq 1$ in $A$.

Proof. Assume the result is false and let $(Q, A)$ be a counterexample for which $|Q|+|A|$ is minimal. Let $|A|=p_{1}^{b_{1}} \ldots p_{k}^{b_{k}}$ be the canonical decomposition of $|A|$ as a product of distinct prime powers. The minimality of $|A|$ forces $b_{1}=\ldots=b_{k}=1$. Let $q$ be a prime divisor of $|Q|$ and let $Q_{0}$ be the Sylow $q$-subgroup of $Q$.

(1) $Q_{0}=Q$. For suppose not. Let $Q_{1}$ be the Hall $q$-complement of $Q$. Let $A_{0}=\left\{a \in A \mid a\right.$ acts trivially on $\left.Q_{0}\right\}$, and let $A=A_{0} \times A_{1}$. Now there exists $v_{0} \in Q_{0}$ such that $v_{0} a_{1} \neq v_{0}$ for any $a_{1} \in A_{1}-\{1\}$ and there exists $v_{1} \in Q_{1}$ such that $v_{1} a_{0} \neq v_{1}$ for any $a_{0} \in A_{0}-\{1\}$. Let $v=v_{0} v_{1}$. Then $v a \neq v$ for any $a \in A-\{1\}$. This establishes (1).

(2) Contradiction. Let $a_{i}$ be an element of $A$ of order $p_{i}$ and let

$$
Q_{1}=\left\{w \in Q \mid w a_{i}=w\right\} \text {. }
$$

Then $Q=\cup Q_{i}$ (set-theoretic union). Let $|Q|=q^{n},\left|Q: Q_{i}\right|=q^{n_{i}}$. Since $a_{i}$ permutes the elements of $Q-Q_{i}$ in to orbits of length $p_{i}, q^{n_{i}} \equiv 1 \bmod p_{i}$ if $p_{i} \neq q$. In particular, $n_{i} \geqq d_{i}$ where $d_{i}$ is the least positive integer for which $q^{d_{i}} \equiv 1 \bmod p_{i}$ if $p_{\imath} \neq q$. But now,

$$
\sum_{i=1}^{k} 1 / q^{n_{i}} \leqq 1 / q+\sum_{p_{i} \neq q} 1 / q^{n_{i}} \leqq 1 / q+\sum_{p_{i} \neq q} 1 / q^{d_{i}}<1
$$


by Lemma 1. On the other hand, the equation $Q=\cup Q_{i}$ implies that

and thus $\sum_{i=1}^{k} 1 / q^{n_{i}} \geqq 1$. The contradiction is established.

$$
\begin{aligned}
& |Q| \leqq \sum_{i=1}^{k}\left|Q_{i}\right| \\
& \text { contradiction is established. }
\end{aligned}
$$

\section{Intersection theorems}

Let $G$ be a finite group and let $A$ be a cyclic subgroup of $G$. Let $|A|=p_{1}^{a_{1}} \ldots p_{k}^{a_{k}}$ where $p_{1}, \ldots, p_{k}$ are distinct primes and $a_{1}, \ldots, a_{k}$ positive integers. Let $A_{i}$ be the subgroup of $A$ of order $p_{i}$ and let $N_{i}$ be the normaliser of $A_{i}$ in $G$. Then $(G, A)$ has property $\left({ }^{*}\right)$ if and only if $G$ is not the set-theoretic union of the groups $N_{1}, \ldots, N_{k}$. Let $\left|G: N_{i}\right|=n_{i}$. We see that $(G, A)$ has property $\left(^{*}\right)$ if $\Sigma 1 / n_{i} \leqq 1$. If $G$ is simple, then $n_{i}>\max \left\{p_{i}\right\}$, so we get

Proposition 1. If $G$ is simple and $A$ is cyclic, then $(G, A)$ has property $\left({ }^{*}\right)$.

Suppose now that $A \cap F(G)=1$. Let $K_{i}$ be the core of $N_{i}$, that is $K_{i}$ is the largest normal subgroup of $G$ contained in $N_{i}$. Since $G / K_{i}$ is a permutation group on $n_{i}$ symbols and $A_{i} \geq K_{i}, n_{i} \geqq p_{i}^{a_{i}}+1$. Lemma 2 implies

Proposition 2. If $A$ is a cyclic subgroup of $G$ such that $A \cap F(G)=1$ and no Sylow subgroup of $A$ has prime order, then $(G, A)$ has property $\left(^{*}\right)$.

Finally we show

Proposition 3. Let $A$ be a cyclic subgroup of a finite group $G$ such that $C_{G}(F(G))$ is abelian. Assume that $A \cap F(G)=1$. Then $(G, A)$ has property $\left({ }^{*}\right)$.

Proof. Since $A \cap F(G)=1$ and $C_{G}(F(G))=Z(F(G)), A$ acts faithfully on $F(G)$ by conjugation. The result follows from Theorem 1.

\section{REFERENCES}

(1) J. S. BRodKey, A note on finite groups with an abelian Sylow subgroup, Proc. Amer. Math. Soc., 14 (1963), 132-133.

(2) M. Herzog, Intersections of nilpotent Hall subgroups, Pacific J. Math. 36 (1971), 331-333.

(3) N. ITo, Über den kleinsten p-Durchschnitt auflösbarer Gruppen, Arch. Math., 9 (1958), 27-32.

\section{UNiversity COLlege \\ DUBLIN \\ IRELAND}

